Tropical Journal of Pharmaceutical Research April 2011; 10 (2): 203-210

(C) Pharmacotherapy Group, Faculty of Pharmacy, University of Benin Benin City, 300001 Nigeria.

All rights reserved.

Research Article

Available online at http://www.tjpr.org

\title{
Niosomal Delivery of Isoniazid - Development and Characterization
}

\author{
Gyanendra Singh ${ }^{1}$, Harinath Dwivedi ${ }^{1}$, Shailendra K Saraf ${ }^{2}$ and \\ Shubhini A Saraf ${ }^{1 *}$ \\ ${ }^{1}$ Department of Pharmaceutics, Faculty of Pharmacy, Babu Banarasi Das National Institute of Technology \& \\ Management, ${ }^{2}$ Faculty of Pharmacy, Northern India Engineering College, Lucknow (Uttar Pradesh), India
}

\begin{abstract}
Purpose: To develop a niosomal formulation for the delivery of isoniazid to achieve effective treatment of tuberculosis.

Methods: Niosomes were prepared by reverse phase evaporation method and given a charge with a charge-inducing agent, dicetyl phosphate. Drug entrapment efficiency in the niosomes was determined spectrophotometrically. The niosomes were further characterized for their particle size, polydispersity index (PI) and zeta potential as well as by scanning electron microscopy and stability studies. Furthermore, in vitro drug release and cellular uptake studies on the niosomes by macrophage J744 A were undertaken.

Results: Suitable isoniazid niosomes were obtained. The niosomes demonstrated a potential to remain in the treated site for prolonged periods and were also capable of maintaining steady drug concentrations for up to $30 \mathrm{~h}$. Cellular uptake of the drug-loaded niosomes by macrophage cells was as high as $61.8 \%$, a level that is capable of achieving effective treatment of tuberculosis.

Conclusion: The isoniazid niosomes developed are capable of reducing drug dose and toxicity as well as dosing frequency which should bring about improved patient compliance. More importantly, macrophage targeting should be feasible at sites where tuberculosis bacteria are harbored.
\end{abstract}

Keywords: Niosome, Macrophage targeting, Isoniazid, Tuberculosis, Drug release, Cellular uptake. 


\section{INTRODUCTION}

Isoniazid (INH) is an effective drug for the treatment of tuberculosis. There is evidence that isoniazid $(\mathrm{INH})$ inhibits the synthesis of mycolic acid, an essential component of bacterial cell wall, and also combines with an enzyme that is uniquely found in strains of mycobacterium. Resistance to INH can occur due to reduction in intracellular penetration of the drug.

Tuberculosis still remains the cause of high mortality and morbidity, particularly in developing countries. It is curable to a large extent but concern about the disease has resurfaced due to the emergence of multidrug resistant strains and the AIDS pandemic [1]. Short-term chemotherapy is associated with many disadvantages such as toxicity, decreased bioavailability at the target site and most important, non-compliance by the patient, thus leading to drug resistance. Some of the shortcomings of chemotherapy can be circumvented by developing a drug delivery system that would release the drug in a sustained manner [2].

The concept of using submicron carrier systems for the delivery of antibiotics has received recognition [3]. For effective chemotherapy, an optimal concentration of therapeutic agents must reach the affected tissue(s) but sometimes, in an attempt to achieve,this, serious side effects may occur. Niosome is a biodegradable, biocompatible and non-immunogenic carrier in which drug can be encapsulated. Encapsulation of bioactive agent in niosome prevents inactivation of the drug, targets the biologically active compound to the targeted tissue and provides slow release of the loaded drug into the circulatory system, thereby reducing its toxicity if effective uptake is achieved [4].

In earlier studies, antitubercular drugs such as INH and rifampicin (RIF) entrapped in PLG polymer when injected as a single dose, resulted in sustained release of the drug for 6 - 7 weeks into various organs of mice. The pharmacokinetics of PLG-encapsulated antitubercular drugs, administered either individually or in combination in mice, has been reported $[5,6]$.

\section{EXPERIMENTAL}

\section{Chemicals and drugs}

Span 60 and Span 20 (sorbitan monostearate, MW 430.6 and 346.5, respectively), cholesterol (MW 386.7), dicetyl phosphate (MW 546.9), chloroform, diethyl ether, and isniazid (INH, MW 137.1) were all obtained from Sigma, St. Louis, USA, while RPMI 1640 nutrient broth was procured from Sigma-Aldrich. All the other reagents used were of analytical grade.

\section{Preparation of niosomes}

Drug-containing niosomes were prepared by reverse phase evaporation (RPE) method, frequently employed for the entrapment of hydrophilic drugs [7], using Span 20 or 60 (SP) as a non-ionic surfactant, cholesterol $(\mathrm{CH})$ as an enhancer of niosomal membrane rigidity and di-cetylphosphate (DCP) as a negative charge inducer [8]. Niosomes with varying ratios of these materials were prepared (see Table 1). They were dissolved $(10 \mathrm{mg} / \mathrm{ml})$ in a chloroform: diethyl ether $(1: 1)$ blend $(10 \mathrm{ml})$ in a $100 \mathrm{ml}$ round bottom flask to form the organic phase. An aqueous phase containing isoniazid $(10 \mathrm{mg} / \mathrm{ml})$ was added, with the ratio of the organic phase to aqueous phase ratio fixed at 5:1. The flask was covered with parafilm to prevent evaporation of the organic phase and then sonicated with an ultrasonic bath sonicator (SN2P, Toschon Industries Pvt Ltd, India) at $50{ }^{0} \mathrm{C}$ for $5 \mathrm{~min}$. A stable white emulsion was formed from which the organic solvents were slowly evaporated at $50{ }^{\circ} \mathrm{C}$ using a rotary vacuum evaporator until a thin film was formed on the walls of the flask. The film was hydrated with $10 \mathrm{ml}$ of phosphate buffer solution (PBS, pH 7.4) to produce an aqueous niosomal suspension containing 100 $\mathrm{mg}$ isoniazid/10ml. This suspension was 
initially kept at $50{ }^{\circ} \mathrm{C}$ in a thermostatted water bath for $1 \mathrm{~h}$ and at room temperature overnight to obtain the drug- niosomes.

\section{Separation of free (un-entrapped) drug}

Free (unentrapped) drug was removed from the niosomes by centrifugation of the dispersion [8] at $14,000 \mathrm{rpm}$ at $4{ }^{\circ} \mathrm{C}$ for 60 $\min$ in a refrigerated centrifuge (3-18 K,Sartorius, AG Weender landstrasse, Gottingen, Germany). The supernatant was removed and the pellet (residue) was resuspended in PBS. This process was repeated twice to ensure that free drug was completely removed.

\section{Particle size determination}

The mean size of the particles was measured by an optical microscope (CH20i BIMF, Olympus India Pvt Ltd) fitted with a camera (Yoko CCD Camera, Taiwan) [7]. The particle size distribution of each formulation was evaluated by determining the size of 100 randomly selected niosomes with the aid of Medical Pro software (version 3.0)

\section{Evaluation of zeta potential and polydispersity index (PI)}

The surface charge of the niosomes was determined by measuring the electrophoretic mobility of the niosomal particles using a zetasizer (MAL 1021384, Malvern Instruments Ltd, UK). Polydispersity index was determined as a measure of homogeneity. Values were obtained from the printed report of Malvern zetasizer which includes the percent intensity in terms of size distribution of niosomes and their respective sizes [8]. Small values of $\mathrm{PI}$ indicate a homogeneous population while high values indicate heterogeneity.

\section{Determination of drug entrapment efficiency}

Entrapment of isoniazid (INH) in the niosomes was determined using a previously reported method $[8,9]$. This method includes separation of unentrapped drug using Sephadex-75 minicolumn and then evaluating the entrapment efficiency by disrupting the vesicles with $0.1 \%$ Triton$\mathrm{X} 100$. The resulting mixture was centrifuged at $3000 \mathrm{rpm}$ for $5 \mathrm{~min}$, with the supernatant was decanted off and suitably diluted with PBS $\mathrm{pH}$ 7.4. The drug was estimated spectrophotometrically (UV-1700, Shimadzu Corporation, Kyoto, Japan) at $261 \mathrm{~nm}$ against PBS containing Triton-X100 as blank. Entrapment efficiency (EE) was determined relative to the original drug concentration according to Eq 1.

$\mathrm{EE}(\%)=(\mathrm{ED} / \mathrm{TD}) 100$

where ED is entrapped drug concentration and TD is theoretical drug concentration (10 $\mathrm{mg} / \mathrm{ml}$ )

\section{Scanning electron microscopy}

The morphology of the niosome was studied by scanning electron microscopy (SEM, Leo430, Cambridge, U.K.). Niosome samples were prepared by air-drying on an aluminum stub. The stubs were then coated with gold to a thickness of 200 to $500 A^{0}$ under argon atmosphere using a gold sputter module in a high vacuum evaporator. The coated samples were randomly scanned by SEM.

\section{In vitro drug release evaluation}

The in vitro drug release profile of the isoniazid niosomal formulations was assessed by a previously reported method [10]. The niosomal formulation was first centrifuged to remove un-entrapped drug. Next, $1 \mathrm{ml}$ of the niosomal sample was packed into a dialysis tube, which in turn was placed in a beaker containing $100 \mathrm{ml}$ of PBS (pH 7.4). The solution containing the dialysis tube (MW cutoff $10,000 \mathrm{Da}$ ) was stirred on a magnetic stirrer at $50 \mathrm{rpm}$ at $37 \pm 1{ }^{\circ} \mathrm{C}$. Samples were withdrawn at various time intervals over a period of $48 \mathrm{~h}$. Each withdrawal was followed by replenishment of the release medium with an equal volume of 
fresh medium to maintain sink conditions. The samples were analyzed spectrophotometrically for drug content at $261 \mathrm{~nm}$ (Shimadzu 1601, Japan). The tests were carried out in triplicate.

\section{Assessment of the physical stability of niosomes}

Samples of niosomes were sealed in $30 \mathrm{ml}$ clear glass vials, stored at 4,30 and $40{ }^{\circ} \mathrm{C}$, respectively, for 90 days and assessed periodically for changes in particle size and residual drug content as previously described.

\section{Establishment and maintenance of cell line}

A mouse macrophage cell line (J744 A.1) was grown as a monolayer using RPMI 1640 media, without glutamine, but supplemented with $10 \%$ fetal bovine serum (for nurturing the cell line). The media were also supplemented with $100 \mu \mathrm{g} / \mathrm{ml}$ each of penicillin and streptomycin, to suppress the growth of microorganisms. The cell lines employed in this study were maintained in a humidified incubator at $37 \pm 2{ }^{\circ} \mathrm{C}$ with 95 and $5 \%$ air and $\mathrm{CO}_{2}$, respectively. Cells were subcultured twice weekly by simply resuspending the cells using 0.2 and $0.025 \%$ trypsin and EDTA, respectively, and then replacing half of the cell suspension with fresh media. Adherent cells were grown to 80 $\%$ confluence in tissue culture grade flasks and then subcultured by discarding the used medium and leaving the cells which adhered to the bottom of the flask.

\section{Viable cell count}

Viable and non-viable cells were distinguished using a haemocytometer. For this purpose, trypan blue was used. Live cells exclude trypan blue stain, leaving them with a normal appearance under the microscope. Dead cells, however take up the stain, making them appear blue. An equal volume of cells and stain $(0.4 \% \mathrm{w} / \mathrm{v})$ were mixed and applied to the bright-line haemocytometer.
The number of cells counted (ignoring blue cells) was multiplied by $2 \times 10^{4}$ to calculate number of viable cells $/ \mathrm{ml}$, thus taking into account the dilution factor upon addition of trypan blue.

\section{Cell uptake studies}

Calcein-loaded niosomes were prepared to study their uptake in J744 A.1 mouse macrophage cells. Plain calcein-loaded niosome were assayed. The calcein-loaded niosomes were prepared by a similar method to that used for the drug-loaded niosomes with calcein substituted for the drug. The concentration of calcein used was $0.5 \mathrm{mg} / \mathrm{ml}$ in PBS. The calcein-loaded niosomes were purified from unloaded calcein niosomes with Sephadex G-75 column.

Calcein-loaded niosomes $(1 \mathrm{ml})$ were diluted in $1 \mathrm{ml}$ RPMl 1640 media and added to monolayers of J744 A.1 cell lines $\left(2 \times 10^{5}\right)$, grown in 96-well culture plate and incubated for $240 \mathrm{~min}$ at $37 \pm 2 \stackrel{\circ}{ } \mathrm{C}$. The cell monolayers were thoroughly washed in PBS and lysed in 0.75 $\mathrm{ml}$ of lysis buffer (PBS containing $0.1 \%$ Triton $X-100)$. These cells were exposed to serial concentrations of the indicated dye (20-0.5 $\mu \mathrm{g} / \mathrm{ml}$ of control formulation and $7.5-0.5$ $\mu \mathrm{g} / \mathrm{ml}$ of coupled formulation in $100 \mu \mathrm{l}$ of culture medium, RPMI 1640 supplemented with $5 \%$ heat-inactivated fetal bovine serum) over a 3-day incubation period at $37{ }^{\circ} \mathrm{C}, 5 \%$ $\mathrm{CO}_{2}$, and $95 \%$ relative humidity.

Their fluorescence was measured in lysis buffer extract using florescence activated cell sorter (FACS, Becton Dickinson, Gurgaon, Hariyana, India) and all the calculations were carried out with Cellquest software (version 5.2, BD Biosciences, Canada).

\section{Fluorescent microscopy}

Cells were cultured in 6-well Petri dish with $1.8 \mathrm{~mm}$ cover slips for $24 \mathrm{~h}$. Calcein-loaded niosomes were added to the cell culture media RPMI 1640 at a concentration of 0.1 $\mathrm{mg} / \mathrm{ml}$. After incubation for $3 \mathrm{~h}$ at $37^{\circ} \mathrm{C}$, the cells were washed six times with PBS. The 
cover slips were put on slides coated with buffered mounting medium and viewed with a Nikon fluorescence microscope (Japan) [14].

\section{Statistical analysis:}

Data, including kinetic data, were processed with MS Excel 2007 and are presented as mean \pm standard deviation.

\section{RESULTS}

Increase in noisome size was observed with increase in cholesterol content. In vitro drug release of RPE-III-60 was best explained by Higuchi equation as the plot showed the highest linearity $\left(r^{2}=0.98\right)$, followed by first order $\left(r^{2}=0.97\right)$ and zero order $\left(r^{2}=0.88\right)$. On the basis of regression coefficient $\left(r^{2}\right)$ and unpaired t-test, the highest $r^{2}$ value was obtained for RPE-III-60. Hence, RPE-III-60 batch was selected for niosomal release experiments. Zeta potential for this batch was $23 \mathrm{mv}$ and while polydispersity index was 0.14 . These values indicate good stability and homogeneity of the formulation. The corresponding $r^{2}(0.98)$ for the KoresmeyerPeppas equation indicate good linearity while SEM revealed that the niosomes were spherical. Niosome formulations were more stable at 4 than at 30 and $40{ }^{\circ} \mathrm{C}(75 \pm 5 \%$ $\mathrm{RH}$ ) (Fig 1-3).

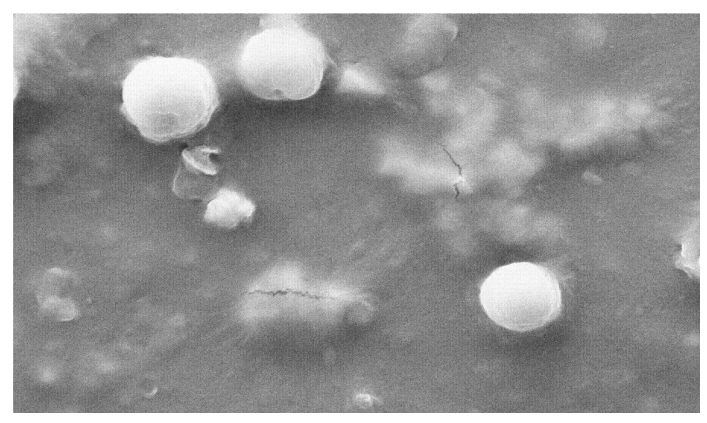

Fig 1: Electron micrographs of isoniazid niosomes (RPE-III-60)

$P$ values for drug release were 0.1578 and 0.6532 , respectively.

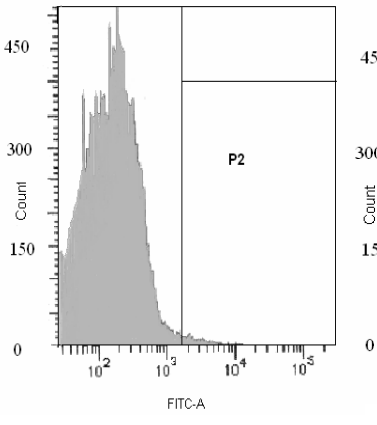

(a)

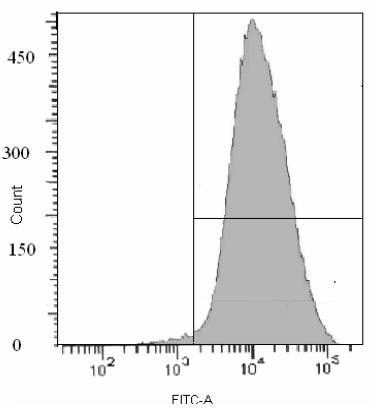

(b)
Fig 2: Autoluminicence of (a) calcein-loaded niosomes ,denoted by P1 peak, and (b) calcein-loaded niosomes absorbed by macrophage cells, denoted by P2 peak

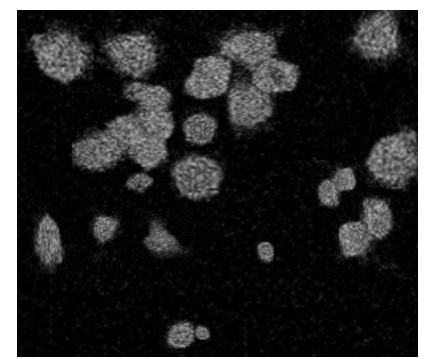

Fig 3: Fluorescence image of isoniazid niosomes coupled with calcein dye following internalization by macrophage cells

A value of $61.8 \%$ was obtained as autoluminiscence of calcein-loaded niosomes absorbed by macrophage cells denoted by P2 peak.

Figure 4 shows that as the proportion of cholesterol in the niosomes increased, particle size, drug entrapment and $t_{50 \%}$ (time taken to attain 50\% drug release) also increased. An earlier study had also found that incorporation of cholesterol in niosomes delayed in vitro drug release [11].

The regression coefficient $\left(r^{2}\right)$ when the in vitro drug release data were subjected to Koresmeyer-Peppas, $1^{\text {st }}$ order, Higuchi and zero order release models yielded 0.98, 0.98, 0.97 and 0.88 , respectively for RPE-III-60. The release exponent, ' $n$ ', for the Koresmeyer-Peppas model was 0.78 , which appears to indicate a coupling of diffusion and erosion mechanisms. This is the so- 
called anomalous diffusion mechanism, which indicates that drug release was controlled by more than one process. A value of $n=0.45$ indicates Fickian diffusion; $0.45<$ $\mathrm{n}<0.89$ indicates anomalous (non-Fickian) diffusion.
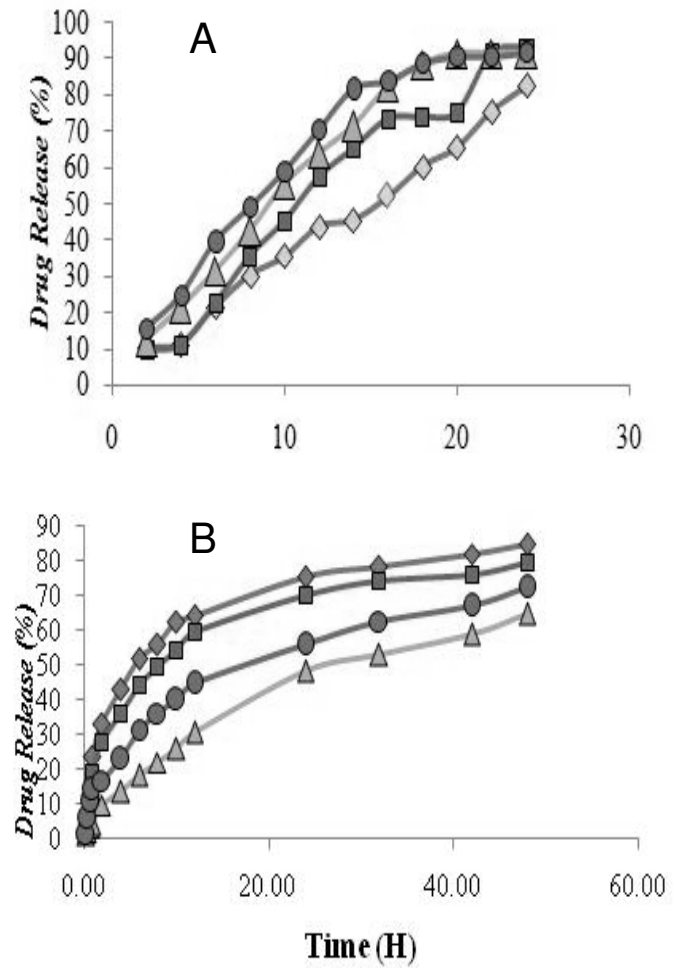

Fig 4: Cumulative release of isoniazid niosomes in (a) simulated gastric fluid $(\mathrm{pH} 1.2)$, (b) simulated intestinal fluid ( $\mathrm{pH} 7.4$ ) where $\bullet=R P E$ III-20 (Batch III containing Span-20); = RPE IV-20 (Batch IV containing Span-20); $\bullet=R P E$ III-60 (Batch III containing Span-60); $\boldsymbol{\Delta}=R P E$ IV-60 (Batch IV containing Span-60)

\section{Cell uptake of niosomes}

Cell uptake data indicate that calcein-loaded niosomes were taken up easily by J744A.1 macrophage cell line to a maximum level of $61.8 \%$ for RPE- III-60.

\section{DISCUSSION}

Increase in particle size was observed upon increasing the cholesterol content.
Entrapment efficiency increased to an optimum level on increasing the cholesterol level and thereafter decreased. Cholesterol $(\mathrm{CH})$ is an important component for changing the fluidity of bilayers of niosomes as the molecules of the former are sandwiched between the molecules of the surfactant (SP) thus rendering the bilayer of the niosomes rigid ${ }^{[9]}$.

On the basis of coefficient of regression $\left(r^{2}\right)$ data, formukation RPE-III-60 was selected as the optimum batch $[12,13]$. On the basis of $r^{2}$ value, RPE-III-60 and RPE-IV-60 had correlation coefficient $\left(R^{2}\right)$ values close to one, i.e., 0.9454 and 0.8621 , respectively (Table 1). The corresponding plot (log cumulative percent drug release vs time) for the Koresmeyer-Peppas model indicate good linearity $\left(r^{2}=0.98\right)$. The release exponent ' $n$ ' was 0.78 , which appears to indicate a coupling of diffusion and erosion mechanisms (or anomalous diffusion) and may indicate that drug release is controlled by more than one process ${ }^{[14]}$.

At optimum SP:CH ratio (6:3), SP bilayers are saturated completely with $\mathrm{CH}$ molecules, hence no further significant increase in vesicle size was observed; however, drug entrapment was enhanced. Similarly, di-cetyl phosphate (DCP) also affected drug entrapment efficiency in the niosomes because its incorporation in the bilayers of the niosome enhances the vesicle size of the niosomes probably due to the identical charge in the bilayers, leading to repulsion in the bilayers and hence increase in size and drug entrapment.

Negligible degradation and leaching was observed at temperature $4{ }^{\circ} \mathrm{C}$ but significant leaching occurred at both 30 and $40{ }^{\circ} \mathrm{C}$, indicating the need to store the niosomes at 4 ${ }^{\circ} \mathrm{C}$.

Zeta potential (ZP) of $23 \mathrm{mv}$ and polydispersity index $(\mathrm{PI})$ of 0.14 for formulation RPEIII-60 shows that the formulation was homogeneous since $\mathrm{PI}>0.3$ is indicative of 
heterogeneity [16]. Ideally, the optimum value of zeta potential should lie between +25 and $25 \mathrm{mv}$ [17]. Outside this range, the niosomes may not remain stable for a long period of time and this could adversely affect parameters such as entrapment efficiency and sustained release. A higher zeta potential indicates higher kinetic energy and tends to move particles towards agglomeration $[17,18]$.

Statistical analysis revealed that there was a significant difference between drug release in simulated gastric and intestinal fluids. On the basis of coefficient of regression $\left(r^{2}\right)$ data and unpaired t-test, RPE-III-60 batch with the highest Koresmeyer-Peppas $r^{2}$ value was staken as the most suitable isoniazid niosomal formulation.

The niosomes obtained in the present work released the entrapped drug at steady rate for a longer duration $(20-30 \mathrm{~h})$ than the $10-$ $15 \mathrm{~h}$ reported for gliclazide niosomes by other researchers [16]. Moreover, the niosomes prepared in our work were comparatively smaller and more homogenous than the gliclazide niosomes.

The fluorescent studies indicate that the isoniazid niosomes are capable of remaining in treated sites in the body for prolonged periods while maintaining constant drug concentration in the process. However, studies to confirm the extent and duration of action of the formulation in vivo were not performed. Nonetheless, the isoniazid niosomal formulation should improve patient compliance as a result of reduced frequency of administration could lead to decreased dose-dependent side effects often associated with repeated administration of conventional isoniazid dosage forms.

\section{CONCLUSION}

Isoniazid niosomal formulations are capable of facilitating reduced drug dose, dose frequency and toxicity when given parenterally or via other suitable routes. Their use could improve patient compliance and most importantly, macrophage targeting would be possible at sites where the mycobacteria responsible for tuberculosis are harbored.

\section{ACKNOWLEDGEMENT}

One of the authors (Gyanendra Singh) wishes to acknowledge a scholarship grant from the All India Council for Technical Education (AICTE) during the course of this work.

\section{REFERENCES}

1. Chretien J. Tuberculosis Today. Eur. Res. J. 1995; 20: 617- 619

2. Eva CW, Ryan T, Norma A. A Novel Technique For Localized Drug Delivery. Bio Phys J 2009; 96: 681- 687.

3. Gursoy A. Liposome Encapsulated antibiotics: physiochemical and antibacterial properties. STP Pharma Science 2000; 10: 285 - 291.

4. Jain $C P, V y a s S P$. Preparation and characterization of niosomes containing rifampicin for lung targeting. J. Microencap 1995; 12: 401-407.

5. Dutt M, Khuller GK. Therapeutic efficacy of poly (DL-Lactide-Co-

Glycolide) encapsulated antitubercular drugs against Mycobacterium tuberculosis infection induced in Mice. Antimicrob. Agents Chemother 2001;

45: 363-366.

6. Roopa Karki, Mamatha GC, Subramanya G, Udupa $N$. Preparation, characterization and tissue disposition of niosomes containing isoniazid. Rasayan J. Chem. 2008; 2: 224-227.

7. Uchegbu IF, Vyas SP. Nonionic surfactant-based vesicles (niosomes) in drug delivery. Int. J. Pharm 1998; 172: 33-70.

8. Elbary A, El-laithy HM, Tadros MI. Sucrose stearatebased proniosome-derived Niosomes for the nebulisable delivery of cromolyn sodium. Int. J. Pharm. 2008; 357: 189-198.

9. Hao Y, Zhao F, Li N, Yang Y, Li K. Studies on a high encapsulation of colchicine by a niosome system. Int. J. Pharm. 2002; 244: 73-80.

10. Jain $C P$, Vyas SP, Dixit VK. Niosomal System for Delivery of Rifampicin to Lymphatics. Int. J. Pharma Sci. 2006; 68: 575 - 578 .

11. Jain $C P$, Vyas $S P$. Preparation and characterization of niosomes containing rifampicin for lung targeting. J. Microencap 1995; 12: 401-407.

12. Desai TR, Finlay WH. Nebulization of Niosomal all trans-retinoic acid: an inexpensive alternative to conventional liposomes. Int. J. Pharm. 2002; 241: $311-317$ 
13. Blazek-Welsh Al, Rhodes DG. A Maltodextrin-based proNiosomes. AAPS PharmSci. 2001; 3: 1-8.

14. Arunothayanun $P$, Bernard MS, Craig DQM. Effect of processing variables on the physical characteristics of non ionic surfactant vesicles (niosomes) formed from a hexadecyle diglycerol ether. Int J. Pharm. 2000; 201: 7-14.

15. Korsmeyer RW, Gurny R, Doelker E, Buri P, Peppas $N A$. Mechanisms of solute release from porous hydrophilic polymers. Int J. Pharm. 1983; 15: 25-35.

16. Raymond MS, Josbert MM, Marcel AF, Andrienne PJ, Grietje M, Gert S. Liposome encapsulated prednisolone phosphate inhibits growth of established tumor in mice. Neoplasia. 2005; 7: $118-127$.

17. Sinko PJ. Martin's Physical Pharmacy and Pharmaceutical Sciences. Edn 5, New York: Lippincott Williams \& Wilkins, 2006; pp 503509.

18. Tamizharasi S, Dubey A, Rathi $V$, Rathi JC. Development and characterization of niosomal drug delivery of gliclazide. J. Young Pharm. 2009; 3: 205-209. 